\title{
Transient amplitude behavior analysis of nonlinear power ultrasonic transducers with application to ultrasonic squeeze film levitation
}

Journal of Intelligent Material Systems and Structures 24(6) 745-752

(c) The Author(s) 2012

Reprints and permissions: sagepub.co.uk/journalsPermissions.nav DOI: I0.I I77// 045389XI2463460 jim.sagepub.com (SSAGE

\author{
Sebastian Mojrzisch and Jörg Wallaschek
}

\begin{abstract}
In this article, force and self-excitation driving methods for ultrasonic transducers are compared with each other in sense of their transient amplitude behavior in the presence of nonlinearities. An equivalent circuit transducer model is simplified to a series oscillator. The simplified model is averaged by the Van der Pol method in order to reduce the system at hand to its amplitude dynamics. The transient amplitude behavior of both driving methods is presented in an analytical form. At high vibration amplitudes, the system's natural frequency varies due to the nonlinear stiffness of the piezoelectric material and the vibration amplitude is likely to break down due to the jump phenomena. Therefore, the averaged models are extended by the nonlinear effects. From the amplitude behavior analysis of both systems, it follows that self-excitation is the preferable driving method in sense of obtaining a high operation bandwidth and a stable oscillation.
\end{abstract}

\section{Keywords}

Control, piezoelectric, actuator resonance, ultrasonic transducer, levitation

\section{Introduction}

In applications that require high precision and stiffness with the absence of stick-slip effects, noncontact bearings are frequently used. Generally, air bearings are the choice for high precision coordinate measuring machines and other nanoscale manufacturing machines. A drawback of air bearings is the need for pressurized air and their low dynamics. Hence, ultrasonic squeeze film levitation bearings offer a promising alternative. With their ability to adjust the levitation gap, stiffness and damping by controlling the vibration amplitude, they form an active bearing with comparable supporting forces to conventional air bearings. Instead of pressurized air that is fed through the porous material into the gap, ultrasonic levitation bearings generate the supporting air film by squeezing the air trapped between two surfaces. The squeeze film effect takes place when one of the two coplanar planes vibrates with a vibration amplitude in the range of the gap distance. In case of ultrasonic squeeze film levitation, the vibration frequency is beyond the human audible range. The highfrequency vibration is usually generated by a piezoelectric ultrasonic transducer. Several ultrasonic bearings have been reported in the literature (Ide et al., 2005;
Oiwa and Kato, 2004; Stolarski, 2007; Wiesendanger, 2001; Zhao et al., 2012; Zhao and Mojrzisch, 2009), but the dynamical properties were not investigated. Oiwa and Kato (2004) presented an active ultrasonic bearing system with a fixed excitation frequency and a Proportional / Integral (PI)-feedback-controller for the levitation gap control. The levitation gap was measured and set according to a reference value by increasing or decreasing the vibration amplitude of the ultrasonic transducer. Oiwa did not use a resonance frequency controller. Thus, his system was driven at small vibration amplitudes and under permanent control of a human operator, who tuned the resonance frequency of the ultrasonic transducer. The focus of Oiwa's work was directed to precise the levitation gap control. However, due to nonlinear and thermal effects in the piezoelectric material and additional load effects in the

Institute of Dynamics and Vibration Research, Leibniz Universität Hannover, Hannover, Germany

\section{Corresponding author:}

Sebastian Mojrzisch, Institute of Dynamics and Vibration Research, Leibniz Universität Hannover, Hannover 30167, Germany.

Email: mojrzisch@ids.uni-hannover.de 
levitation gap, such a driving technique is not suited for high bandwidth ultrasonic squeeze film levitation bearings.

This article focuses on the transient amplitude behavior of the power ultrasonic transducers with a high Qfactor. A high Q-factor, which indicates a low damping, is needed as the system's losses are mainly induced by damping in the ultrasonic transducer. However, a high Q-factor leads to a long settling time of the vibration amplitude, whereas a short settling time is desired. These two design objectives lead to a trade-off that cannot be solved by control strategies used in power ultrasonics.

This article is structured as follows: After an introduction to ultrasonic levitation and pointing out the difference to other ultrasonic processes, a common transducer model is simplified to its mechanical components. This is valid due to the assumption of excitation close to the resonance frequency. The obtained simplified model is reduced by averaging methods to its transient amplitude behavior. It is shown that in case of self-excitation, the system's transient amplitude response always shows first-order lag type. In case of force excitation, the transient response is of secondorder lag type and shows more complex behavior in the presence of nonlinearities.

\section{Ultrasonic squeeze film levitation}

The ultrasonic squeeze film levitation is based on highfrequency vibration of a piston or a plate close to a surface. By repetitive compression and decompression, the air trapped in the gap is squeezed. As a consequence, the so-called squeeze film is generated in the gap. The levitated object is supported by this squeeze film. A basic ultrasonic levitation squeeze film-bearing setup is shown in Figure 1.

In case of large dimensions of the vibrating member and the surface compared to the gap distance, the lateral boundaries can be neglected and the air in the gap can be treated as trapped. For such a system, Chu and Apfel (1982) gave a formulation to calculate the radiation pressure that was used (Hashimoto et al., 1996; Ueha et al., 2000) to calculate the levitation force of ultrasonic squeeze film levitation bearings. Chu and Apfel presented an expression for Rayleigh radiation pressure in ideal gas on a perfectly reflecting target

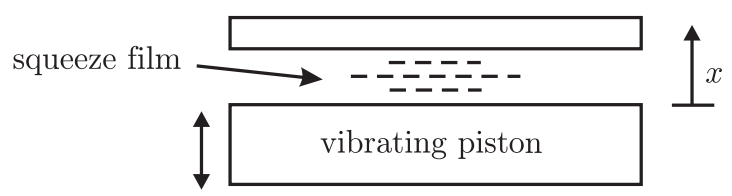

Figure I. Schematic illustration of ultrasonic squeeze film levitation.

$$
\begin{gathered}
\langle p\rangle=\left\langle p_{\mathrm{T}}-p_{0}\right\rangle=\frac{1+\gamma}{2}\langle E\rangle[1+\operatorname{sinc}(2 k x)] \\
\langle E\rangle=\frac{a_{0}^{2}}{4} \cdot \frac{\rho_{0} \omega^{2}}{\sin ^{2}(k x)} ; \quad k=\frac{\omega}{c} .
\end{gathered}
$$

The definition of each symbol is as follows: $\langle p\rangle$ is the time-averaged radiation pressure, $p_{T}$ is the pressure acting on target, $p_{0}$ is the ambient pressure, $\langle E\rangle$ is the time-averaged energy density, $\gamma$ is the specific heat ratio of the medium, $\rho_{0}$ is the density of the medium, $k$ is the wave number, $\omega$ is the angular frequency of the wave, $c$ is the speed of sound, $a_{0}$ is the vibration amplitude, and $x$ is the distance between the two surfaces (gap). Assuming that the gap is in the dimensions of several micrometers $x \ll 1$ the argument $k x$ is small and the sinc-function can be assumed as equal to one. Furthermore, for small arguments of the sin-function, the expression is linearized. Thus, equation (1) can be rewritten in the following form

$$
\langle p\rangle=\frac{1+\gamma}{4} \rho_{0} c^{2} \frac{a_{0}^{2}}{x^{2}} .
$$

The validity of equation (3) has been experimentally proven for piston and flexural vibrators (Hashimoto et al., 1996; Ueha et al., 2000). In equation (3), the pressure depends on the quotient of the vibration amplitude and the gap distance. Here, the vibration amplitude means the positive peak value of the high-frequency harmonic vibration. Therefore, in the following only the vibration amplitude should be investigated neglecting the high-frequency vibration signal.

In order to realize a position feedback control, a linear relation between the vibration amplitude and the levitation force acting on the levitated object has to be found. Therefore, equation (3) is linearized around an operating point. It should be noted that in the region of resonance, there is a fixed ratio between the current flowing into the transducer and its vibration amplitude: $i_{a}=\alpha \omega_{0} a_{0}$, where $i_{a}$ is the current amplitude and $\alpha$ is the proportional factor (see Figure 4)

$$
f(x, i)=\left.\frac{\partial\langle p\rangle \cdot A}{\partial x_{0}}\right|_{\mathrm{OP}} \cdot x+\left.\frac{\partial\langle p\rangle \cdot A}{\partial i_{0}}\right|_{\mathrm{OP}} \cdot i_{a}=k_{x} x+k_{i} i_{a} .
$$

Since the vibration amplitude is proportional to the current amplitude, it is sufficient to measure the current and to derive the vibration amplitude.

Figure 2 shows the linearized open-loop model of the levitation system. The equivalent mass of the levitated object is represented by $k_{\mathrm{m}}$. The constants $k_{\mathrm{i}}$ and $k_{\mathrm{x}}$ are derived from equation (3) by equation (4). These two constants depend on the levitation surface $A$ and the operating point. The damping constant $k_{\mathrm{d}}$ is obtained from Hagen-Poiseuille law (Griffin et al., 1966) for a given geometry or has to be determined 


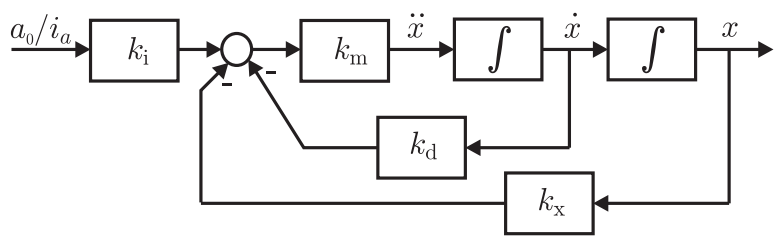

Figure 2. Linearized system model of the ultrasonic levitation bearing.

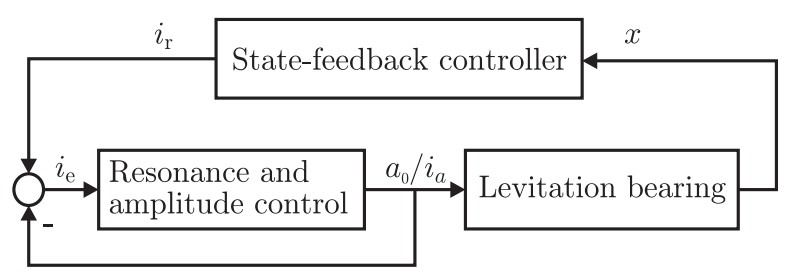

Figure 3. Overall control system.

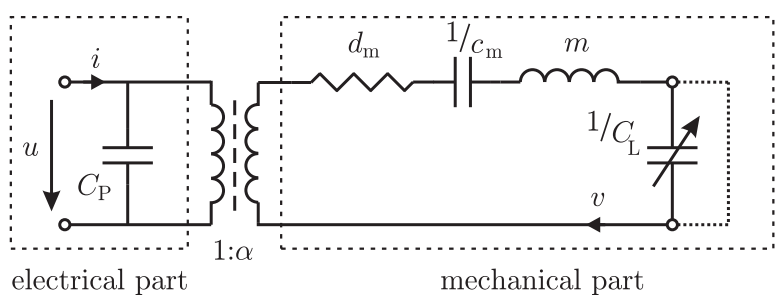

Figure 4. Equivalent model of an ultrasonic transducer.

experimentally. Figure 3 shows the overall control loop. The control of the bearing parameters is performed by state-feedback control. Its design is a straightforward procedure and is not been considered here in detail. The main interest concerns the resonance and amplitude control block, which is intended for tuning the right vibration frequency and setting up the vibration amplitude according to the reference current $i_{\mathrm{r}}$. As $i_{\mathrm{r}}$ is slowly changing with time compared to the ultrasonic frequency signal, the reference current is modulated onto the ultrasonic frequency signal. The resulting vibration amplitude of the ultrasonic transducer $a_{0}$ is also slowly changing with time. Thus, the high-frequency ultrasonic signal is averaged in order to investigate the two driving methods and their transient response.

In contrast to the system studied in this article, a short settling time of vibration amplitude is not of great importance for most of the ultrasonic processes, because the systems are damped by the process, and the vibration amplitude is not aimed to be changed rapidly. In contrast to this, an active ultrasonic squeeze film levitation bearing is aimed to allow fast change of the bearing stiffness, damping, and levitation gap. In Figure 2, the bearing's input is given by the vibration amplitude or current amplitude. For the system at hand, changing the input is the only way to influence the output. Therefore, a high vibration amplitude bandwidth and a stable oscillation are desirable.

\section{Modeling of the piezoelectric transducer}

In high-power ultrasonic applications, the transducer is driven close to its resonance to make use of the amplitude magnification factor. For linear systems, the magnification factor is equal to the quality factor $\mathrm{Q}=(2 \zeta)^{-1}$ ( $\zeta$ is the damping ratio), which is typically higher than 1000 for Langevin transducers radiating in air. From the energetic point of view, the Q-factor should be as high as possible to reduce losses in the transducer, but a high Q-factor also results in a long settling time. Thus, weakly damped transducers show low-amplitude dynamics. This is undesirable as the levitation force is dependent on the vibration amplitude, what means, that the levitation force cannot change faster than the vibration amplitude is changing.

As already mentioned, the scope of this article is directed to the transient vibration amplitude behavior of ultrasonic transducers. Thus, the equivalent model shown in Figure 4 is simplified to get rid of components out of scope. For the given system in Figure 4, the admittance transfer function is defined as follows, with the mass $m$, stiffness $c_{\mathrm{m}}$, damper $d_{\mathrm{m}}$, and the parallel capacitance $C_{\mathrm{P}}$

$$
\begin{aligned}
& Y(j \omega)=\frac{I(j \omega)}{U(j \omega)}=Y_{\mathrm{el}}(\mathrm{j} \omega)+Y_{\text {mech }}(\mathrm{j} \omega) \\
& Y(\mathrm{j} \omega)=\mathrm{j} \omega C_{\mathrm{P}}+\frac{\mathrm{j} \omega \frac{\alpha^{2}}{m}}{-\omega^{2}+\frac{d_{\mathrm{m}}}{m} \mathrm{j} \omega+\frac{c_{\mathrm{m}}}{m}} .
\end{aligned}
$$

The load compliance $C_{\mathrm{L}}$ is absent in equation (6) when the gap distance is kept constant. A constant gap distance is a natural assumption in position control of a reference gap value. In transient case, for example, during the start up process of the bearing, the load compliance $C_{\mathrm{L}}$ is considered as a part of $c_{\mathrm{m}}$. The damping that results from an air operation of the transducer is added to the transducer's damping. Thus, the electrical path across $C_{\mathrm{L}}$ is short-circuited and the mechanical part forms a series oscillator. The admittance for low and high frequencies is governed by the first right-hand term in equation (6); the second right-hand term becomes only relevant when $\omega$ approaches resonance of the series oscillator. As only the region close to resonance is of interest, $Y_{\mathrm{el}}$ will be dropped. It adds linearly with the frequency increasing component, which has no influence on the systems behavior for small frequency changes around the resonance in high Q-factor systems. Furthermore, equation (6) is divided by $j \omega$. This results in a transfer function between charge and voltage, in which $C_{\mathrm{P}}$ can be subtracted from the real part. The resulting system is a series oscillator that describes the 
Table I. Identified parameters for the used ultrasonic transducer.

\begin{tabular}{lll}
\hline Dimension & Value & Unit \\
\hline $\mathrm{m}$ & 0.227 & $\mathrm{~kg}$ \\
$c_{\mathrm{m}}$ & $3.66 \times 10^{9}$ & $\mathrm{~N} / \mathrm{m}$ \\
$d_{\mathrm{m}}$ & 20.29 & $\Omega$ \\
$\alpha$ & 2.38 & $\mathrm{~N} / \mathrm{V}$ \\
$\mathrm{Q}_{\mathrm{m}}$ & 1420 & \\
$f_{0}$ & 20209 & $\mathrm{~Hz}$ \\
\hline
\end{tabular}

a)

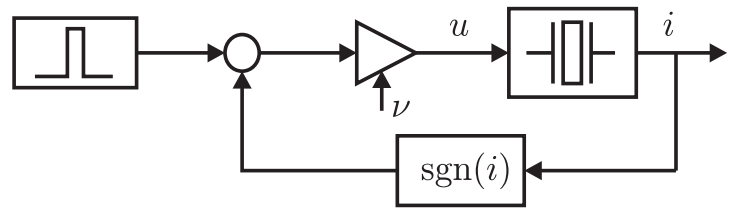

b)

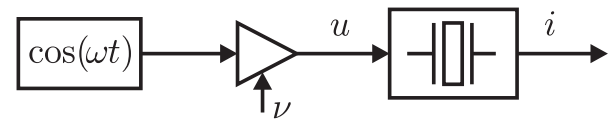

Figure 5. (a) Self-excitation driving method and (b) force excitation driving method.

transducers behavior close to its mechanical resonance. In the "Transient response of vibration amplitude" section, the transient response for an ultrasonic transducer is calculated. The presented step responses of self- and force-excited systems are calculated using the equivalent model parameters listed in Table 1.

\section{Transient response of vibration amplitude}

With the simplifications made above, the transient response can be calculated for the self- and forceexcited ultrasonic transducers. The two excitation methods are shown in Figure 5. In the upper part, a self-excitation configuration is presented; this is also called autoresonant or self-resonant (Babitsky et al., 2004; Babitsky and Sokolov, 2007). A band-pass filter can be added into the positive-feedback loop in order to select the desired resonance frequency or to add a phase shift to the feedback. As an initial condition is needed to start oscillation, the circuit needs an impulse (disturbance) in the beginning. After this, the dissipated energy in the transducer is delivered by the amplifier and its amplification factor is set by $v$. Due to the positive feedback of the current that is proportional to the vibration velocity, current and voltage are in phase and the system is in resonance. To obtain stable limit cycle oscillation, the feedback is limited to one by a sgnfunction block. This prevents oscillation to become infinite due to the positive feedback. The lower part of Figure 5 shows the force excitation. In this schematic illustration, the frequency tuning circuit is not shown.
But as excitation close to resonance is desired, the excitation frequency has to be tuned, for example, by a phase-locked loop (PLL) (see Ramos-Fernandez et al., 1985). This is typically performed by a phase comparison of the current and the voltage signals. For both configurations, the amplitude is modulated by setting the amplification factor $\nu$ according to the reference signal $i_{\mathrm{r}}$.

Since mechanical displacement is proportional to electrical charge, charge is used to formulate the differential equations for the considered excitation methods. The equations of motion for the systems shown in Figure 5 are as follows

$$
\begin{aligned}
& \ddot{q}+2 \zeta \omega_{0} \dot{q}+\omega_{0}^{2} q=v \operatorname{sgn}(\dot{q}) \\
& \ddot{q}+2 \zeta \omega_{0} \dot{q}+\omega_{0}^{2} q=v \cos \omega t .
\end{aligned}
$$

The difference between both systems is the kind of excitation on the right-hand side of equations (7) and (8). Because the slowly changing amplitude of $q$ is of interest, the equations are averaged. For averaging of equation (7), the method of slowly changing amplitude presented in Magnus and Popp (1997) is used as this is applicable to free and self-excited vibration. This method is a simplified version of the Van der Pol method (1927) that is used to average equation (8).

In the method of slowly changing amplitude, the amplitude is considered as a function of time

$$
\begin{gathered}
q=b(t) \cos \omega t \\
\dot{q}=-b \omega \sin \omega t \\
\ddot{q}=-2 \dot{b} \omega \sin \omega t-b \omega^{2} \cos \omega t .
\end{gathered}
$$

As $b(t)$ is slowly changing with time, its derivates are small and can be neglected in the derivates of $q$. The sgn-function is approximated by its first harmonic of Fourier series

$$
\nu \operatorname{sgn}(\dot{q}) \approx-\frac{4 v}{\pi} \sin \omega t
$$

After substituting equations (9) to (12) into equation (7), the $\sin \omega t$ and the $\cos \omega t$ terms are equated separately to zero. The equation containing $\sin \omega t$ terms describes the amplitude behavior. Since the positive current feedback can be phase shifted by an additional filter, the more general case is approximated by

$$
\nu \operatorname{sgn}(\dot{q}) \approx-\frac{4 \nu}{\pi} \sin (\omega t+\varphi)
$$

Using equation (13) and equating terms containing $\sin \omega t$ to zero give for small values of $\varphi$

$$
\dot{b}+\zeta \omega_{0} b=\frac{2 \nu}{\pi \omega} \cos \varphi .
$$


This is a differential equation of first order with the solution for $b$

$$
b=c_{1} \cdot \cos \varphi\left(1-e^{-\zeta \omega_{0} t}\right) \quad c_{1}=\frac{2 v}{\zeta \omega_{0} \pi \omega} .
$$

Since $v$ is arbitrary, $c_{1}$ can be set equal to one. For zero phase lag in the feedback loop, also $\cos \varphi$ becomes equal to 1 , and equation (15) simplifies to

$$
\hat{q}=b=1-e^{-\zeta \omega_{0} t} .
$$

It is remarkable that the self-excited configuration always shows first-order lag amplitude behavior. That means even if the positive feedback is phase shifted, the transient response is of first-order lag type. This is of great importance for the control of the vibration amplitude and the whole ultrasonic levitation process, because the proportional gain of the amplitude controller can be set to high values without risk of amplitude oscillations. Thus, the amplitude settling time can be reduced, and this results in an improved dynamic characteristic, respectively.

As a numerical example, the settling time till $90 \%$ for the ultrasonic transducer given in Table 1 with $\mathrm{Q}_{\mathrm{m}}=1420$ is $50 \mathrm{~ms}$. The transient amplitude behavior from equation (16) is shown in Figure 6 for different Q-factors. In this figure, the system's input is scaled up for lower Q-factors in order to get the same steadystate amplitude. For a higher value of $\mathrm{Q}$, the settling time of the amplitude increases proportionally to the Q-factor.

The above calculation is made for the excitation case that means positive impedance at the output of the amplifier. Furthermore, it is also possible to actively damp the transducer vibration by inverting the sgnfunction in the feedback, which results in a negative impedance output of the amplifier. This leads to a phase shift of $180^{\circ}$ between voltage and current, and thus, the current is flowing back from the transducer to

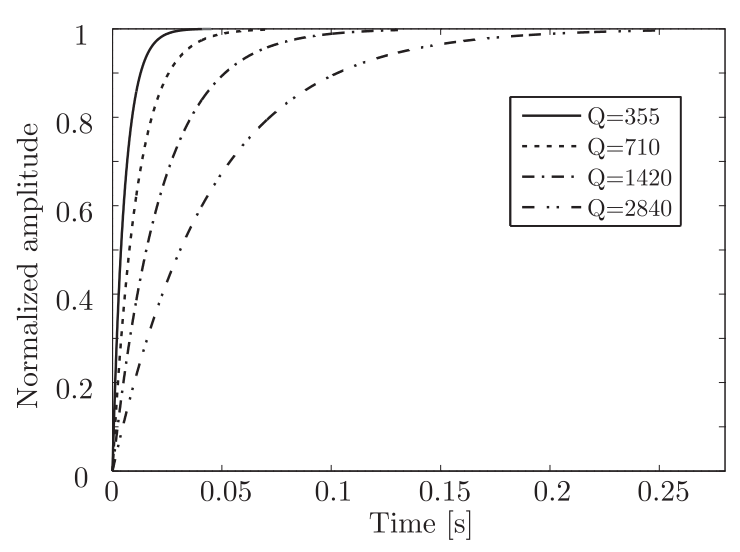

Figure 6. Amplitude step response in a self-excited system for different values of Q-factor. the amplifier. This is relevant since the vibration amplitude decreases with the same rate as it increases. Note that in the force-excited configuration, an inversion of the excitation signal leads to an unstable behavior because of phase detection problems and beats that occur due to wrongly tuned excitation frequency.

The terms containing $\cos \omega t$ describe the change in vibration frequency. For the linear case without phase shift shown above, the vibration frequency is equal to $\omega_{0}$. By introducing phase shift to the positive feedback, the vibration frequency changes according to

$$
\omega^{2}=\omega_{0}^{2}+\frac{4 v}{\pi b} \sin \varphi .
$$

The piezoelectric material is known to exhibit a nonlinear behavior. This mainly appears as generation of second and third harmonics (Aurelle et al., 1996). In order to show the influence of nonlinearities, the stiffness $c_{\mathrm{m}}$ is assumed to be nonlinear and to have a restoring force to displacement characteristic of Duffing type, thus a cubic term multiplied by the factor $\beta$ is added. A cubic restoring force generates the third harmonic

$$
\begin{gathered}
f(q)=\omega_{0}^{2}\left(q+\beta q^{3}\right) \\
q^{3} \approx \frac{3}{4} b^{2}(b \cos \omega t) .
\end{gathered}
$$

As a consequence, a term is added to the $\cos \omega t$ terms, which is approximated by its first harmonic component, as shown in equation (19). Therefore, equation (17) is extended by a parabolic vibration frequency dependence on the vibration amplitude. This is well known for Duffing oscillators, but it should be noted that the nonlinearity basically influences the vibration frequency and not the transient amplitude behavior

$$
\omega^{2}=\omega_{0}^{2}\left(1+\frac{3}{4} \beta b^{2}\right)+\frac{4 \nu}{\pi b} \sin \varphi .
$$

In the force-excited systems, the Duffing characteristics can lead to the jump phenomena. For the selfexcited systems, the jump phenomenon does not take place as the relation between phase and amplitude is unique for the considered system (Sokolov and Babitsky, 2001).

The second system in Figure 5, described by equation (8), is averaged by the Van der Pol method, which uses the following expressions to calculate the amplitude behavior

$$
\begin{gathered}
q=b_{1} \sin \omega t+b_{2} \cos \omega t \\
\dot{q}=b_{1} \omega \cos \omega t-b_{2} \omega \sin \omega t \\
\ddot{q}=\left(2 \dot{b}_{1} \omega-b_{2} \omega^{2}\right) \cos \omega t-\left(2 \dot{b}_{2} \omega+b_{1} \omega^{2}\right) \sin \omega t .
\end{gathered}
$$

Here, the higher derivates of $b_{1}$ and $b_{2}$ have also been neglected. Equations (21) to (23) are substituted 


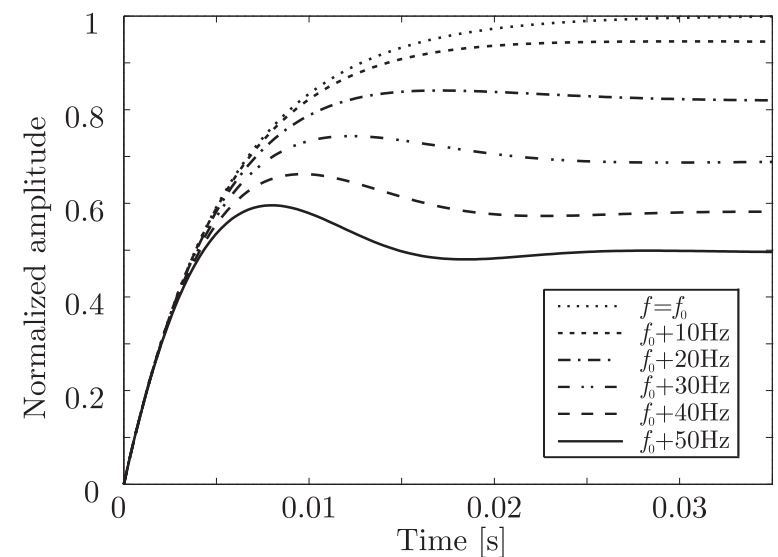

(a) $Q_{\mathrm{m}}=355$

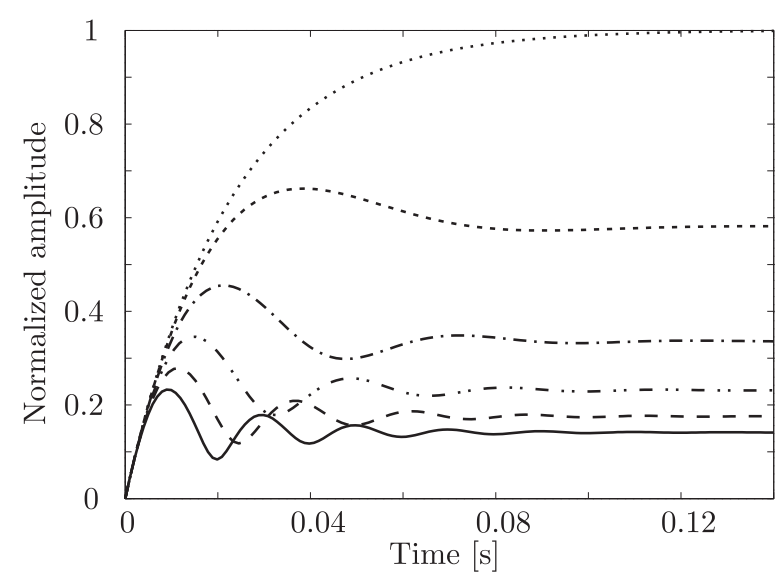

(c) $Q_{\mathrm{m}}=1420$

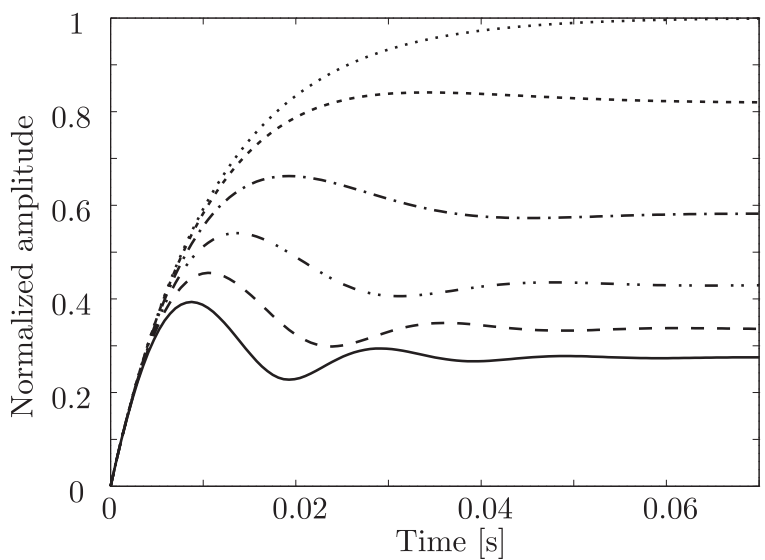

(b) $Q_{\mathrm{m}}=710$

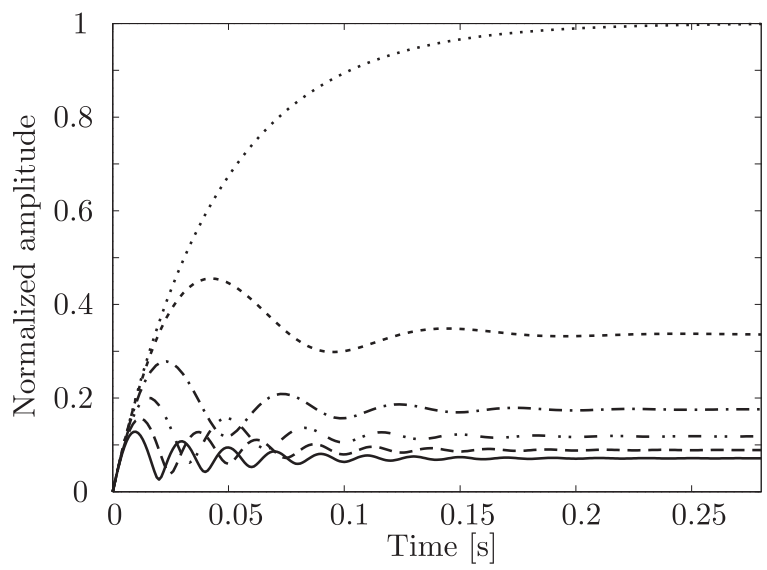

(d) $Q_{\mathrm{m}}=2840$

Figure 7. Amplitude step response in a force-excited system for different values of the Q-factor.

into equation (8) and equated separately for terms containing $\sin \omega t$ and $\cos \omega t$ to zero. This results in the following system

$$
\left(\begin{array}{l}
\dot{b}_{1} \\
\dot{b}_{2}
\end{array}\right)=\left[\begin{array}{cc}
-\zeta \omega_{0} & -\frac{\omega_{0}^{2}-\omega^{2}}{2 \omega} \\
\frac{\omega_{0}^{2}-\omega^{2}}{2 \omega} & -\zeta \omega_{0}
\end{array}\right]\left(\begin{array}{l}
b_{1} \\
b_{2}
\end{array}\right)+\frac{v}{2 \omega}\left(\begin{array}{l}
1 \\
0
\end{array}\right) u .
$$

When $\omega$ is close to the resonance frequency, the following simplification can be made

$$
\frac{\omega_{0}^{2}-\omega^{2}}{2 \omega} \approx \omega_{0}-\omega .
$$

The averaged amplitude is calculated as the absolute value of $b_{1}$ and $b_{2}$. Besides this, the phase $\psi$ between the excitation and displacement is calculated as shown below

$$
\hat{q}=\sqrt{b_{1}^{2}+b_{2}^{2}}
$$

$$
\psi=\arctan \frac{b_{1}}{b_{2}} .
$$

The step responses for the system in equation (24) are plotted for different Q-factors and excitation frequencies in Figure 7. For an excitation frequency equal to the system's resonance frequency, the system shows the same transient behavior as the self-excited system in Figure 6 . When the excitation frequency is not equal to the resonance frequency, the step response changes. Due to mistuned excitation frequency, beats that occur decay with the time constant of the system (see equation (16)). This leads to amplitude overshot before reaching the steady state. In Figure 7(a) to (d), this behavior is more critical for a high Q-factor system because the amplitude overshot and the beat frequency are higher for the same deviation from the resonance frequency.

By introducing a nonlinear restoring force behavior, the dynamics matrix becomes time variant and dependent on the vibration amplitude. This leads to equation (28) with an amplitude-dependent natural frequency $\eta_{\mathrm{b}}$ as follows 


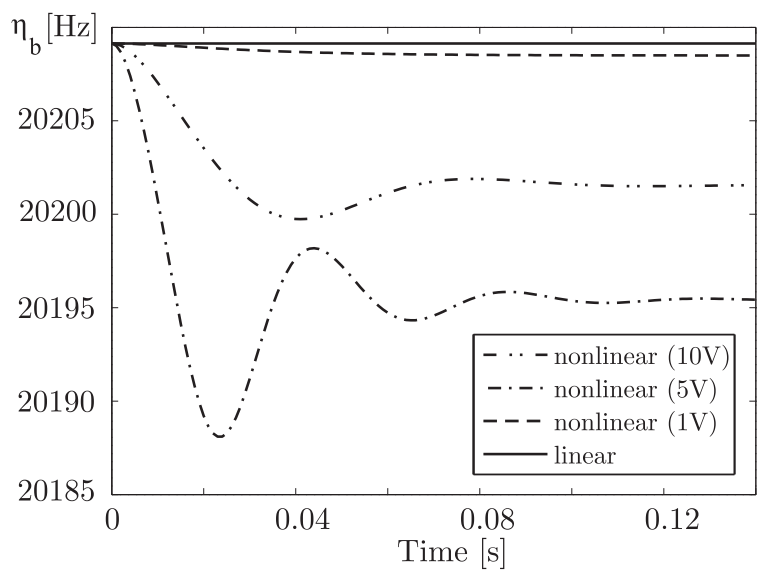

(a) Natural frequency change induced by nonlinearity

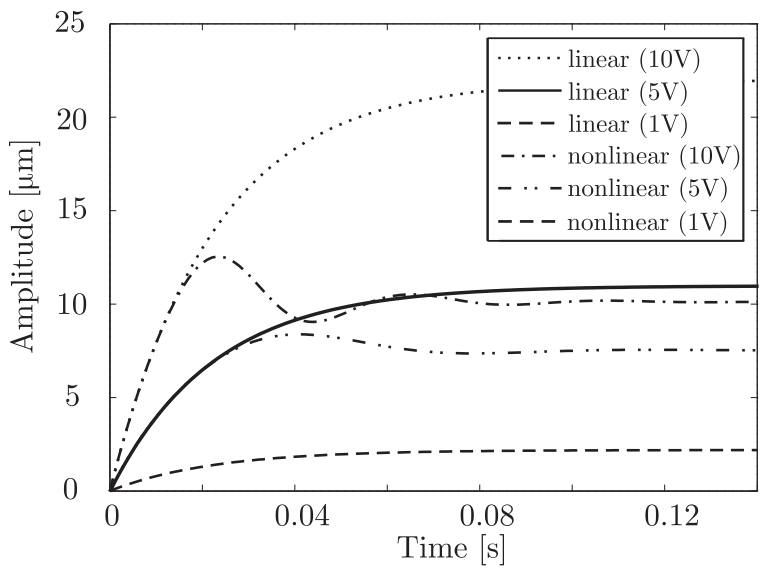

(b) Amplitude response for different excitation levels

Figure 8. Amplitude step responses for a nonlinear ultrasonic transducer, $Q_{\mathrm{m}}=1420, \omega=\omega_{0}, \beta=-1 \times 10^{8} \mathrm{~m}^{-2}$.

$$
\begin{gathered}
\left(\begin{array}{l}
\dot{b}_{1} \\
\dot{b}_{2}
\end{array}\right)=\left[\begin{array}{cc}
-\zeta \omega_{0} & -\frac{\eta_{b}^{2}-\omega^{2}}{2 \omega} \\
\frac{\eta_{b}^{2}-\omega^{2}}{2 \omega} & -\zeta \omega_{0}
\end{array}\right]\left(\begin{array}{l}
b_{1} \\
b_{2}
\end{array}\right)+\frac{v}{2 \omega}\left(\begin{array}{l}
1 \\
0
\end{array}\right) u \\
q^{3} \approx \frac{3}{4}\left(b_{1}^{2}+b_{2}^{2}\right)\left(b_{1} \sin \omega t+b_{2} \cos \omega t\right) \\
\eta_{b}^{2}=\omega_{0}^{2}\left(1+\frac{3}{4} \beta\left(b_{1}^{2}+b_{2}^{2}\right)\right) .
\end{gathered}
$$

The nonlinearity causes a reduction of natural vibration frequency with increasing vibration amplitude. Figure 8(a) depicts the change in natural vibration frequency for different excitation voltages for an excitation in the resonance frequency $\omega_{0}$. Due to beats that occur between the excitation and natural frequencies, the amplitude shows oscillation before reaching the steady state. Since the natural frequency depends on the vibration amplitude, it also shows oscillation during transients. The responses in Figure 8(b) show similar behavior to the responses in Figure 7, whereas in the presence of nonlinearities, even excitation in resonance shows amplitude oscillation for high vibration amplitudes. Besides this, the jump phenomena are likely to happen as during every amplitude change the system's natural frequency also changes. When the jump phenomena take place, the vibration amplitude breaks down. For small vibration amplitudes, the nonlinear system shows close to linear behavior since $\beta \cdot \hat{q}^{2}$ is small.

The behavior described above is critical in sense of a high-amplitude controller gain as the closed-loop system can get unstable. Since the amplitude controller acts on amplitude, equations (24) and (28) can be used in control loop design of PLL systems to calculate the stability margins for a control parameter set.

\section{Conclusion}

The active ultrasonic squeeze film levitation bearings offer a promising solution for a new kind of actively controlled noncontact bearing systems. As the force generated in the levitation gap is dependent on vibration amplitude, its control is of special interest. It turns out that the self-excitation is the preferable driving method in sense of resonance and amplitude control. It does not show the jump phenomena, and its settling behavior is of first-order lag type. This makes it possible to set the Pgain in the amplitude feedback controller to high values and reduces the slow settling time for the low-damped ultrasonic transducers. Furthermore, it is possible to actively damp the ultrasonic transducer by inverting the sgn-function in the feedback loop of the self-excitation. Force excitation shows amplitude oscillation when the excitation frequency is not equal to the system's natural frequency. Since in the presence of nonlinearities, the natural frequency is influenced by beats in the vibration amplitude, the simultaneous control of the vibration amplitude and the vibration frequency is challenging. As a consequence, it can be concluded that the self-excited driving of ultrasonic transducers is the preferable method to make ultrasonic levitation bearings robust and fast.

\section{Funding}

This research received no specific grant from any funding agency in the public, commercial, or not-for-profit sectors.

\section{References}

Aurelle N, Guyomar D, Richard C, et al. (1996) Nonlinear behavior of an ultrasonic transducer. Ultrasonics 34(5): 187-191.

Babitsky V and Sokolov I (2007) Autoresonant hemostat concept for engineering application of nonlinear vibration modes. Nonlinear Dynamics 50: 447-460. 
Babitsky V, Kalashnikov A and Molodtsov F (2004) Autoresonant control of ultrasonically assisted cutting. Mechatronics 14: 91-114.

Chu B and Apfel R (1982) Acoustic radiation pressure produced by a beam of sound. Journal of the Acoustical Society of America 72(6): 1673-1687.

Griffin W, Richardson H and Yamanami S (1966) A study of fluid squeeze-film damping. Journal of Basic Engineering 88: 451-456.

Hashimoto Y, Koike Y and Ueha S (1996) Near-field acoustic levitation of planar specimens using flexural vibration. Journal of the Acoustical Society of America 100(4): 2057-2061.

Ide T, Friend J, Nakamura K, et al. (2005) A low-profile design for the noncontact ultrasonically levitated stage. Japanese Journal of Applied Physics 44(2005): 4662-4665.

Magnus K and Popp K (1997) Schwingungen. Stuttgart: Teubner.

Oiwa T and Kato M (2004) Squeeze air bearing based on ultrasonic oscillation: motion error compensation using amplitude modulation. Review of Scientific Instruments 75(11): 4615-4621.

Ramos-Fernandez A, Montoya-Vitini F and Gallego-Juarez J (1985) Automatic system for dynamic control of resonance in high power and high Q ultrasonic transducers. Ultrasonics 23(4): 151-156.
Sokolov I and Babitsky V (2001) Phase control of selfsustained vibration. Journal of Sound and Vibration 248(4): 725-744.

Stolarski T (2007) Performance of self-lifting linear air contact. Journal of Mechanical Engineering Science 1(221): 1103-1115.

Ueha S, Hashimoto Y and Koike Y (2000) Non-contact transportation using near-field acoustic levitation. Ultrasonics 38(8): 26-32.

Van der Pol B (1927) Forced oscillations in a circuit with non-linear resistance. Philosophical Magazine Series 7(3): 65-80.

Wiesendanger M (2001) Squeeze film air bearings using piezoelectric bending elements. PhD Thesis, Ecole Polytechnique Federale de Lausanne, Lausane. DOI: 10.5075/epfl-thesis-2336.

Zhao S and Mojrzisch S (2009) Development of an active squeeze film journal bearing using high power ultrasonic transducers. In: Conference on smart materials, adaptive structures and intelligent systems (SMASIS2009), pp. 195-202: SMASIS2009SMASIS1244. September 21-23, ASME. Avaliable at: http:// dx.doi.org/10.1115/SMASIS2009-1244

Zhao S, Mojrzisch S and Wallaschek J (2012) An ultrasonic levitation journal bearing able to control spindle center position. Mechanical Systems and Signal Processing. Available at: http://dx.doi.org/10.1016/j.ymssp.2012.05.006 